\title{
SÍNTOMAS SOMÁTICOS MÉDICAMENTE INEXPLICADOS
}

\author{
Rafael Fernández* \\ Concepción Fernández** \\ Isaac AmIgo"* \\ Marino Pérez**
}

Unidad de Salud Mental del Salnés. Complexo Hospitalario de Pontevedra*

Departamento de Paicologla. Universided de Oviedo**

\section{RESUMEN}

Se realiza una revisión de los principales factores influyentes en los denominados sintomas y sindromes somáticos médicamente inexplicados. Estos factores se presentan conforme a su presumible modo de influencia para to que se dividen en factores implicados en la predisposición, en el inicio y en la persistencia. Sobre la base de la presentación anterior se discuten tres posibles vias de desamollo de los problemas de somatización. En concreto, 1) aquella en la que predomina la dimensión de regulación de relaciones interpersonales, 2) la preocupación hipocondríca y 3) los déficits en la discriminación de los estados emocionales.

\section{Palabras clave: SOMATZACIÓN, SINTOMAS FUNCIONALES, TRASTOA- NOS SOMATOFORMES.}

\section{SUMMARY}

In this paper a review of the main factors associated with the unexplained somatic syndromes is made. These factors are

Correspondencla: Concepción Femández. Universidad de Oviedo. Dpto. de Paicología. Plaza Feljoo, s/n. 33003. Oviedo. E-mail: frconcha uniovi.es 
divided according its influence way in the predisposition, onset and maintaining of the somatic problems. Three ways in the development of the somatization problems are discussed a) regulation of the interpersonal relationship, b) hypochondriacal worry and c) deficits in the discrimination of the emotional states.

Key words: SOMATIZATION, FUNCTIONAL SYMPTOMS, SOMATOFORM DISORDERS.

\section{INTRODUCCIÓN}

Las molestias somáticas benignas y transitorias son extraordinariamente frecuentes en la población general. En la mayoría de pacientes que llegan a consultar por síntomas para los que no se encuentra una causa médica objetivable, éstos tienden a remitir en un plazo breve. Sin embargo, en una minoría persisten durante mucho tiempo (Stone et al., 2003). Además del costo personal en términos de malestar, limitación funcional y, por tanto, afectación en la calidad de vida, los problemas de somatización pueden tener también una importante repercusión en el gasto sanitario (Barsky et al, 2001).

Estos síntomas, también denominados atípicos, funcionales o somatoformes (Kroenke, 2001) recorren todo el campo de la Medicina (Tabla 1).

Tabla 1. Sintomas o sindromes mødicamente Inexplicados (Kroonke, 2001)

\begin{tabular}{|l|c|}
\hline Especialidad Médica & Shroma o Sindrome Inexplicado Medicamente \\
\hline - Gastroenterologia & - Síndrome del intestino irritable \\
- OTL & - Globo faringeo, disfonfa psicógena \\
- Neurologia & - Cefalea, mareos, síndromes seudoneurológicos \\
- Medicina interna & - Sindrome de fatiga crónica \\
- Reumatología & - Fibromialgia \\
- Odontología & - Sindrome de disfunción mandibular \\
- Cardiología & - Dolor torácico no-cardiaco \\
- Ginecología & - Dolor pélvico crónico \\
\hline
\end{tabular}


Las clasificaciones psicopatológicas incluyen la categoría de los trastornos somatoformes que recoge aquellas condiciones que comparten como caracteristica central la presencia de síntomas somáticos sin explicación médica. Muchos de los pacientes diagnosticados con trastomos somatoformes conforme a la clasificación psicopatológica serán clasificados en otras especialidades médicas de otros modos (por ej. FM en reumatología o Sil en GE) (Caballero y Caballero, 2004).

Hay estudios que documentan el elevado solapamiento entre los síntomas de los pacientes con estos síndromes que, además comparten otras características: mayor prevalencia en mujeres, asociación con trastomos emocionales o sufrimiento psicológico general (más allá del que genera la experiencia continuada de síntomas), conductas y creencias acerca de la condición, mecanismos fisiológicos comunes asf como formas de intervención clínica y respuesta a las mismas (Allen et al, 2002; Barsky y Borus, 1999). Además, se ha observado una elevada "movilidad" entre las características somatoformes cuando se estudian longitudinalmente (Lieb et al, 2002).

Se puede concebir un continuo que va desde síntomas somáticos funcionales transitorios, sin repercusión significativa en la vida diaria $y$, por tanto, sin relevancla clínica, a condiciones crónicas y altamente perturbadoras del funcionamiento diario (Katon, Lin, Von Korff, Russo, Lipscomb y Buss, 1991).

Estos problemas han atraído la atención de los clínicos desde hace mucho. Por ejemplo, Briquet definió el síndrome que lleva su nombre en 1859 y Beard en 1869 describió la neurastenia. Este interés también se reflejó en la inclusión de las escalas de Hipocondría (que pese a su nombre consta de un conjunto de malestares físicos) $\theta$ Histeria en el test psicométrico MMPI.

El calíficativo "inexplicados" aplicado a estos síntomas no quiere decir "inexplicables" o "imaginarios". Como señalan Kirmayer et al (2004) el "salto" entre fisiología, sensaciones y experiencia somática refleja la complejidad del Sistema Nervioso Central y sus procesos que trasladan la perturbación fisiológica en experiencia. Dicha complejidad se refleja en el énfasis de los modelos actuales de los problemas somatoformes en la interacción de procesos cognitivos y perceptivos con cambios conductuales, afectivos y biológicos (Rief y Barsky, 2005). 
El objetivo central es realizar una revisión (necesariamente selectiva dada la amplitud del campo) y síntesis de los principales factores impulsores de la experiencia y persistencia de síntomas médicamente inexplicados crónicos y discapacitantes. Para ello la exposición se ordenará conforme al esquema general: 1) Factores de Predisposición, 2) Factores Desencadenantes y c) Factores de Mantenimiento

\section{FACTORES INFLUYENTES EN LOS SÍNTOMAS MÉDICAMENTE INEXPLICADOS}

\section{Factores de Predisposiclón}

\section{Factores sociodemográflcos}

Los diferentes estudios coinciden en que las mujeres informan con más frecuencia de este tipo de síntomas lo que se ha explicado sobre la base de diferencias biológicas, cognitivas, de socialización y roles sociales asi como mayor exposición a experiencias de abuso y trauma. Otros factores sociodemográficos mencionados son la edad, (a mayor edad mayor información de síntomas), estado civil (los solteros más que los casados), ocupación (desempleados más que los laboralmente activos) y estatus socioeconómico (estatus socioeconómico bajo se asocia a más información) (Pennebaker, 1982).

\section{Factores biológicos}

Estudios de adopción, como el de Cloninger et al. (1984) han encontrado que el trastorno por somatización es cinco veces más frecuente en mujeres familiares en primer grado de pacientes con somatización que en la población general. No obstante, los datos obtenidos en otras investigaciones no apoyan la existencia de influencia genética en los mismos. Torgersen (1986) aunque encontró una concordancia algo mayor entre gemelos monocigóticos que en los dicigóticos $(29 \%$ frente a $10 \%)$, ésta no era significativa. Por lo demás, entre los monocigóticos habla mayor puntuación en un índice de experiencias compartidas en la infancia lo cual bien podría explicar la mayor concordancia. 
El neuroticismo o afectividad negativa, entendido como una dimensión amplia de personalidad relacionada con la tendencia a experimentar emocionalidad negativa, y que se considera como un factor de vulnerabilidad general al trastorno emocional y a la presencia de molestias físicas diversas (Costa y McCrae, 1985), ha sido vinculado directamente con factores genéticos (Watson, 1999).

\section{Factores socloculturales}

Como señalan Katon, Kleinman y Rosen (1982) "la cultura proporciona creencias sobre la enfermedad, visiones sobre como funciona el cuerpo, metáforas dominantes e idiomas que organizan la interpretación del cambio corporal". Kirmayer y Young (1998) enfatizan la dimensión comunicativa de los sintomas corporales a los que se refieren como un "lenguaje del sufrimiento" inteligible culturalmente. Un ejemplo es el del "distrés cardiaco" entre los iraníes. Los síntomas de dicha manifestación son comprendidos como una manera de comunicación prescrita culturalmente acerca de una variedad de preocupaciones, especialmente las que tienen que ver con la pérdida y el pesar. $O$ en la Medicina tradicional coreana los órganos corporales se relacionan simbólicamente con estados emocionales. Asl, los pulmones se relacionan con la preocupación, tristeza y falta de ánimos, el hígado con la ira o los riñones con el miedo (Bhugra y Mastogianni, 2004).

Las diferentes prevalencias en los problemas de somatización entre países apoyarían el papel de los factores socioculturales en la experiencia e informe de síntomas somáticos (Gureje, 2004).

Finalmente, la medicalización (entendida como la invocación de un diagnóstico médico para explicar el malestar flsico no causado por una enfermedad y la aplicación de una intervención médica para tratarlo) que se da en la cultura occidental contemporánea favorece la amplificación, preocupación y consulta por síntomas que en otras épocas no habrían sido objeto de atención especial (Barsky y Borus, 1995).

Experiencias vitales antecedentes de los sintomas somatoformes

En la literatura psicológica y psiquiátrica se destacan dos clases de experiencias como antecedentes remotos significativos de los 
síntomas somatoformes en los adultos. Por una parte, las que tienen que ver con circunstancias que implican alguna forma de victimización o trauma; y, por otra, aquellas relacionadas con la enfermedad y conducta de enfermedad en la familia. Las primeras se entenderían como influencias generales en la medida que se asocian a una mayor probabilidad de desajuste psicológico a lo largo de la vida, mientras que las segundas se podrían contemplar como factores de predisposición más específicos para los problemas de somatización.

\section{Victimización en la infancia}

Experiencias altamente perturbadoras como son las distintas formas de abuso a lo largo de la infancia son informadas con una frecuencla significativamente mayor en los adultos con síndromes somáticos médicamente inexplicados como se pone de relieve en numerosos estudios en los que se ha investigado esta cuestión (Katon, Sullivan y Walter, 2001).

Una limitación metodológica de estos estudios es su carácter retrospectivo. Como indican Lesserman et al. (1996), "es posible que sesgos en el recuerdo afecten o amplifiquen la relación (abusosíntomas somáticos), de modo que aquellos que es más fácil que recuerden abusos sean tarnbién los que informen de mayor distrés psicológico y conductas de enfermedad". También se debe tener en cuenta la posibilidad de que la relación con la somatización se base en terceras variables como pueden ser las dinámicas familiares patológicas o condiciones de pobreza, más probables en contextos en que se dan experiencias de abuso.

Otro problema metodológico son los posibles sesgos en la formación de las muestras. Es posible que éstas no sean representativas de la población de sujetos con historia de abuso. Por ejemplo, en muestras de pacientes de contextos médicos pueden estar sobrerepresentados aquellos con historia de abuso con más problemas de salud y psicológicos, inflándose así esa relación.

El único estudio prospectivo que examinó la posible relación de la experiencia de dolor crónico inexplicado médicamente con experiencias de abuso documentado en la infancia (físico, sexual y negligencia en los cuidados) no confirmó dicha asociación (Raphael, Widom y Lange, 
2001). Aunque en este estudio se evitaron las limitaciones metodológicas derivadas de los estudios retrospectivos no se han dejado de señalar otras limitaciones y sesgos que impiden obtener conclusiones firmes.

Experiencias relacionadas con la salud a lo largo de la infancia

Circunstancias como la convivencia con familiares con enfermedades graves, con conductas de enfermedad intensas y repetidas, la focalización excesiva sobre el estado de salud del hijo o la obtención de refuerzos por parte de éste a través de la conducta de enfermedad, se podrían entender como influencias remotas importantes de los problemas de somatización e hipocondrla del adulto (Benjamin y Emison, 1992).

Estos estudios comparten también las limitaciones que se derivan de los informes retrospectivos. Es posible que, además, los pacientes con problemas somatoformes estén más orientados, por la naturaleza del problema, a recordar e informar de circunstancias que son consistentes con su habitual experiencia de malestar corporal.

No obstante, en el único estudio prospectivo del que se tiene conocimiento (Hotopf, Mayou, Wadsworth y Wessely, 1999) se observó una fuerte relación entre la experiencia de múltiples síntomas físicos médicamente inexplicados a la edad de 36 años y problemas de salud en los padres a la edad de 15; relación que se mantuvo tras controlar la presencia de trastomos psicológicos. No se observó relación entre la experiencia de enfermedades en la infancia y síntomas en la adultez, no obstante, el dolor abdoiminal recurrente en la infancia, que no implicaba la presencia de una enfermedad clara, sl se asoció a este resultado en el seguimiento.

\section{Factores desencadenantes}

\section{Circunstanclas y acontecimlentos vitales negat/vos actuales}

Además de otros síntomas (por ejemplo, afectación del estado de ánimo, ansiedad o perturbaciones del sueño), las molestias corporales son una manifestación habitual en el contexto de condiciones vitales adversas (Fink, 1996) que incluirlan tanto sucesos vitales negativos mayores (por ejemplo, pérdida afectiva) como adversidades 
menores pero crónicas de la vida (por ejemplo, relación mañtal conflictiva). Numerosos estudios han examinado el impacto de una amplia variedad de acontecimientos y circunstancias vitales estresantes. En la revisión de estudios en atención primaria de Katon, Ries y Kleinman (1984) se encontraron prevalencias entre el 25 al $75 \%$ de síntomas directamente relacionados con distrés psicosocial.

\section{Estados emoclonales negat/vos}

Los síntomas emocionales, muchas veces con inicio en el contexto de circunstancias vitales adversas identificables, se asocian a la experiencia de molestias no explicadas médicamente. De hecho, se ha señalado que la somatización es el modo más común de presentación de los problemas psicologicos (Murphy, 1989). La probabilidad de cumplir los criterios diagnósticos de algún trastomo emocional se incrementa linealmente con el número de síntomas (Kisely, Gollberg y Simon, 1997). Por otra parte, los diagnósticos psicopatológicos más comunes en atención primaria de los pacientes con estos síntomas, son los trastornos de adaptación, de ansiedad y depresivos, más que los somatoformes (Bass y Murphy, 1995). En el estudio de Lobo et al (1996) en España, por ejemplo, del grupo de pacientes con síntomas médicamente inexplicados solo el $9,5 \%$ cumplían los criterios diagnósticos del trastomo de somatización y solo el $27 \%$ fueron clasificados en la categoría más amplia de trastornos somatoformes. La mayoría de los pacientes se incluían en el grupo de pacientes con trastomos de ansiedad y depresivos. Como señalan los autores "la somatización es un fenómeno mucho más amplio que el reflejado por categorlas tales como trastomos somatoformes de las clasificaciones diagnósticas oficiales".

La asociación con los trastomos emocionales ha sido repetidamente documentada (Katon y Walker, 1998).

\section{Factores de mantenImlento}

\section{La focallzación somátlca}

Ya el siglo XIX Holland (cit. Lipowski, 1988) se refirió al papel de la atención sobre la percepción corporal del modo siguiente: "entre 
los fenómenos que atestiguan las diversas influencias de la mente sobre los órganos corporales, ¿se ha reparado suficientemente en los efectos que dependen del simple acto de concentrar la atención sobre ellos?"

A través de la atención se hacen más notorias sensaciones que de otra manera pasarlan desapercibidas. Por ejemplo, Pennebaker (1982) instruyó a sujetos a prestar atención a la sensación de bloqueo nasal mientras que a otros se les dio la instrucción de que se fijaran en como el aire pasaba libremente. Los primeros informaron significativamente de más dificultades respiratorias.

Hay evidencia experimental reciente del sesgo atencional de los pacientes somatoformes hacia estímulos relacionados con la corporalidad (Lim y Kim, 2005).

Igualmente las intervenciones psicológicas dirigidas a promover cambios en la focalización corporal parecen tener un efecto terapéutico sobre el malestar somático asl como sobre las preocupaciones y conductas abiertas relacionadas en pacientes con alta ansiedad por la salud (Papageorgiou y Wells, 1998).

\section{Atribuclones}

El significado atribuido a las sensaciones y sintomas corporales influye en la experiencia y la persistencia de las mismas. La importancia de ello ha sido reconocida desde hace mucho como indica la cita de Blackmore. "ideas terribles, formadas solo en la imaginación, afectarán al cerebro y al cuerpo con dolorosas sensaciones" (Blackmore, 1725 cit. Berrios, 2001).

La valoración, no justificada, de sensaciones corporales como señales de enfermedades graves se observa habitualmente en pacientes con distintos problemas somatoformes y se ha señalado como un factor clave en su ocurrencia y persistencia (Salkovskis y Warwick, 2001).

No solo las atribuciones influirlan en la experiencia y continuidad de los síntomas. Hay pruebas de su relevancia también en los cambios fisiológicos mismos. Por ejemplo Browlec, Leventhal y Balaban (1992) encuentran que los sujetos con tendencia a la hipervigilancia y preocupación por el funcionamiento corporal mostraban una mayor 
reactividad autonómica en tareas que implicaban la imaginación de escenas de enfermedad presentadas auditivamente.

En la base de las atribuciones de enfermedad puede haber creencias o reglas personales como considerar incompatible la experiencia de molestias corporales y estar en buen estado de salud (Barsky et al, 1993) o relativas a una supuesta mayor vulnerabilidad a la enfermedad (Salkovskis, 1988). Estas creencias pueden favorecer la experiencia de ansiedad ante molestias físicas con un efecto amplificador de las mismas.

\section{Probiemas emocionales}

Además de la asociación transversal de problemas psicológicos y malestar físico señalada en el apartado dedicado a los factores desencadenantes, la presencia de trastomos psicológicos parece ser también predictor del mantenimiento de los síntomas físicos. Por ejemplo, en el estudio de seguimiento (media de 15 meses) de Speckens, Van Hemert, Bolk, Rooijmans y Hengeveld (1996) hasta un $65 \%$ de los pacientes con síntomas físicos médicamente no explicados y algún trastorno emocional continuaban experimentando los síntomas en el seguimiento. En el estudio de Kooiman et al (2004) la experiencia de ansiedad y depresión (evaluadas con el HAD), la hipocondria (evaluada con el WI) así como la duración de los síntomas en la evaluación inicial predijo su persistencia (seguimiento de 61 semanas de media).

\section{Procesos de aprendizaje}

Determinadas consecuencias asociadas a las conductas de enfermedad, tales como la obtención de atenciones especiales o la evitación de obligaciones penosas podrían tener un efecto reforzador de las mismas aumentando la probabilidad de que se den en distintos contextos.

También se ha señalado su posible funcionalidad en la justificación de fracasos en la vida y, por tanto, en la protección de la autoestima (Kowalski, 2002), en la evitación del estigma asociado al diagnóstico psiquiátrico (Looper y Kirmayer, 2004), en la regulación de relaciones 
interpersonales (Griffith, Griffith y Slovik, 1989), en la comunicación indirecta de malestar (Stuart y Noyes, 1999) o en la evitación de la contemplación y afrontamiento de conflictos y problemas de naturaleza personal o derivados de condiciones sociales particulares (Kirmayer y Young, 1998). Estos procesos de aprendizaje que se pueden dar en los problemas de somatización deben diferenciarse de la simulación y de los trastornos facticios. En estos dos casos se da un intento deliberado de simular o exagerar síntomas para alcanzar determinados fines.

Por otra parte, los acontecimientos vitales que desencadenan una activación fisiológica intensa pueden conducir a la adquisición de respuestas fisiológicas condicionadas ante estímulos que evocan la experiencia. Este proceso de aprendizaje se refleja en la observación frecuente del inicio de trastornos funcionales en el contexto de un acontecimiento relativamente menor que evoca un trauma o circunstancia vital perturbadora del pasado (Reuber et al, 2005).

Finalmente, se debe hacer referencia a procesos de aprendizaje verbal que pueden tener especial relevancia en estos problemas. Los pensamientos $\theta$ imágenes relacionados con la enfermedad con alta carga emocional (por ejemplo, pensar en uno mismo aislado en un hospital y en un estado terminal) podrían entenderse como experiencias de condicionamiento semántico adícionales. Del mismo modo que el sujeto aprende a evitar situaciones externas evocadoras de malestar emocional por sus efectos inmediatos de liberación del mismo, podría implicarse en intentos encubiertos de evitación o supresión de esas experiencias internas desagradables. También los intentos de evitación experiencial pueden verse reforzados por la liberación transitoria del malestar (Wilson y Luciano, 2002).

\section{Cambios conductuales}

Cambios de conducta en respuesta a la enfermedad adaptativos en un primer momento pueden, si se hacen habituales, conducir a un empeoramiento del estado físico general y continuidad de las molestias a la larga. Por ejemplo, la evitación de la actividad que se asocia a intensificación del dolor, tras la recuperación del problema físico que cursó con dolor, puede continuar alentando la percepción 
de mala salud y la presencia de síntomas relacionados con la inactividad. Otras conductas como las comprobaciones corporales para asegurarse de la ausencla de alteraciones fisicas puede llegar a inducir, sl tal conducta es insistente, molestias en la zona corporal objeto de comprobación.

Otros cambios conductuales pueden tener efectos menos directos. Por ejemplo, la reducción de actividades gratificantes influye negativamente en el estado de ánimo lo que en si mismo favorece la experiencia de síntomas por la disminución del umbral de percepción sensorial.

Finalmente, los cambios en la conducta asociados a la asunción del rol de enfermo pueden inducir cambios ambientales con efecto perpetuador. Barsky y Borus (1999) al respecto señalan que "una vez que la persona es etiquetada como enferma es visto y tratado de modos que hacen más difícil la recuperación: se espera de la persona continuidad de la enfermedad $y$, asl, los síntomas persisten".

\section{Infuencias latrogénicas}

Al margen de la influencia de un toco biomédico exclusivo que ensombrece otras influencias posibles (Rosendal y Fink, 2005), las comunicaciones médicas poco claras, la experiencia de errores diagnósticos, efectos secundarios de los tratamientos médicos, hallazgos de anomalias menores tras investigaclones repetidas en las que el paciente se implica en su ánimo de descartar la patologla que teme o comunicaciones contradictorias entre los médicos a los que consulta, son algunas de las posibles consecuencias desafortunadas del contacto con los servicios de salud (Mayou y Famer, 2002).

La actitud médica en ocasiones negativa ante el paciente somatizador se puede transmitir de modo más o menos explicito a éste. La consecuencia pueden ser esfuerzos de demostración abierta de la realidad de los síntomas y del sufrimiento para obtener la respuesta médica deseada (Epstein, Quill y McWhitney, 1999).

\section{DISCusión}

Los distintos factores a los que nos hemos referido separadamente a lo largo de este trabajo operan de manera integrada. En este 
sentido, por ejemplo, Kirmayer y Young (1998) proponen un modelo de la somatización en el que se incluyen factores fisiológicos, psicológicos, interpersonales y socioculturales que contribuyen a los círculos viciosos de la amplificación que da lugar a síntomas médicamente inexplicados discapacitantes (círculos viciosos como los que se dan entre la catastrofización de síntomas y su amplificación, la evitación de actividades y el descondicionamiento físico o la asunción del rol de enfermo que lleva a dificultades interpersonales que inducen perturbación emocional).

También recientemente Rief y Barsky (2005) han propuesto un modelo de los síntomas somatoformes en el que se recogen los principales elementos implicados en su desarrollo y mantenimiento. En este modelo se destaca el papel del sistema nervioso en el filtrado de las sensaciones fisicas en condiciones de normalidad. Sin embargo, condiciones como el estrés crónico y sus consecuencias fisiológicas, el descondicionamiento físico o procesos de sensibilización física provocan un aumento de las señales corporales, por una parte. Por otra parte, factores como la atención selectiva, la ansiedad por el estado de salud o estados emocionales negativos disminuyen el filtraje con un incremento consecuente de la experiencia somática.

En esta revisión nos hemos centrado en los factores psicológicos implicados en el origen y perpetuación del malestar somático crónico médicamente inexplicado. No obstante, hay que destacar que, como señala Kellner (1990), la exclusión de enfermedad no es equiparable a la ausencia de causas físicas en la base de las molestias. En palabras del autor: "puede ser difícil y a veces imposible apreciar una causa física a través de los medios diagnósticos convencionales sin el uso de pruebas que pueden ser de interés para la investigación pero que no se usan rutinariamente en la práctica médica".

Algunos ejemplos de mecanismos fisiológicos inductores de síntomas son la hiperventilación que se ha implicado, por ejemplo, en el dolor torácico no cardiaco (Serlie, Duivenvoorden, Passchier, Ten Cate, Deckers y Erdman, 1996); la afectación del sueño que puede dar lugar a sintomas de dolor osteomuscular similares a los de la fibromialgia (Friedberg y Jason, 2001); el estado de tensión de determinados grupos musculares en cefaleas y otros síndromes dolorosos o el arousal sostenido derivado de la exposición a circunstancias 
estresantes que ocasiona camblos en el sistema inmune (Dantzer, 2005; Rief y Barsky, 2005) o lleva a una "sobrecarga" que se concreta en cambios como disfunciones neuroendocrinas y en la neurotransmisión o sensibilización de las vias espinales y centrales del dolor (Overmier, 2002). Las consecuencias neurobiológicas, además, podrían ser a largo plazo lo que tiene relevancia en la explicación de la vulnerabilidad de los sujetos que han sufrido abusos en la infancia. Por ejemplo, Van Houdenhove y Egle (2001) señalan que el estrés severo en la infancia puede estar en la base de malestar somático de la adultez a través de factores como la hipervigilancia sensorial y sensibilización de mecanismos centrales de procesamiento del dolor.

Existe cierta arbitrariedad en la clasificación propuesta de los factores influyentes. Por ejemplo, la experiencia de enfermedades graves en familiares en la infancia puede entenderse como factor que predispone a los problemas somatoformes en la adultez pero también podría ser un factor desencadenante de conductas de enfermedad en la infancia. Así, Hotopf, Mayou, Wadsworth y Wessely (1999) citan estudios, dentro del campo de la pediatría, que revelan una alta frecuencia de enfermedad cardiaca en padres de niños que acuden al pediatra con quejas de dolor en el área torácica. Del mismo modo, un bajo umbral perceptivo tanto podrla clasificarse como factor de predisposición o de mantenimiento, en la medida que favorece tanto el inicio como la continuidad de molestias físicas y preocupaciones.

Se debe señalar que la conceptualización de algunos de los factores no es inequívoca. Por ejemplo, en relación con la afectividad negativa, se ha entendido tanto como un rasgo general de personalidad con base genética (Watson, 1999) como una tendencia a responder con emocionalidad negativa a una amplia variedad de estímulos relacionada con histonias personales de aprendizaje particulares (Staats, 1996/1997).

Hay divergencias, asimismo, sobre los modos de inducción de síntomas. Por ejemplo, las experiencias vitales traumáticas pueden suponer el inicio de una trayectoria marcada por el malestar somático y emocional. Uno de los posibles factores que dificultan el ajuste a largo plazo podrían ser los intentos desadaptativos de evitación de estímulos externos $e$ internos evocadores de la experiencia tal y 
como han resaltado teóricos psicodinámicos (Horowitz, 1988), cognitivos (Williams, Stiles y Sapiro, 1999) y conductistas (Hayes, Wilson y Strosahl, 1996). El fracaso en la asimilación se ha relacionado, alternativamente, con las consecuencias neuropsicológicas de la exposición al estrés grave. En todo caso, se resalte la evitación condicionada o los cambios neuropsicológicos, en lo que sí parece haber acuerdo es que las disfunciones en el proceso normal de asimilación o la imposibilidad de elaborar una "narrativa" coherente del acontecimiento traumático y sus consecuencias (Waitzkin y Magraña, 1997) puede ser un mecanismo importante en los procesos de somatización.

Un aspecto que merece comentario es la inclusión dentro del apartado dedicado a los procesos de aprendizaje de funciones de la somatización que han sido también resaltadas por teóricos psicodinámicos. Tal es el caso de la somatización como modo de proteger la autoestima, como defensa frente a sentimientos de culpa o como justificación de la evitación de determinadas responsabilidades o compromisos. Aunque ambas corrientes de pensamiento de la psicopatologla partan de supuestos diferentes, el planteamiento es consistente también con el conductista que pone el énfasis en el aprendizaje de modos de afrontamiento (conductas operantes, se podria decir) en base a su funcionalidad ante circunstancias problemáticas de la vida como conflictos, frustraciones, decepciones y cambios (Marino Pérez, 2004).

Merece también destacarse la perspectiva del desarrollo en el acercamiento a los problemas somatoformes. Recientemente varios autores han adoptado este marco e informado de la estrecha asociación de estos problemas con pautas disfuncionales de conducta características de trastornos de la personalidad (Kirmayer, Robbins y Paris, 1994) asi como con estilos característicos en los patrones de apego (Schmidt et al, 2002; Ciechanowski et al, 2002). Lo que se quiere resaltar es que los problemas de somatización pueden basarse en actitudes y pautas aprendidas a lo largo del desarrollo tales como el aprendizaje de que las necesidades de apego solo son cubiertas al expresar malestar somático 0 , alternativamente, el aprendizaje a dirigir la atención aprensivamente sobre el funcionamiento corporal sobre la base de historias de enfermedad 
en la familia. Aunque compartan la tendencia somatizadora, estos dos patrones pueden diferir claramente tanto en su expresión conductual como en los factores desencadenantes y de mantenimiento principales. Así, en el paciente en el que predominan las sospechas hipocondriacas, la enfermedad o muerte de algún allegado o una mayor cobertura en los medios de comunicación a alguna enfermedad puede, por una parte, desencadenar una intensificación de las preocupaciones $y$, por otra parte, determinadas conductas como la búsqueda de reaseguramiento médico y de comprobación corporal repetitivas impedir una atenuación progresiva de la ansiedad y síntomas relacionados. En pacientes con el primer patrón, por el contrario, la experiencia de malestar somático puede iniciarse en el contexto de amenaza a relaciones afectivas frágiles y tener una funcionalidad en la prevención de la posible pérdida.

Otro patrón posible podría derivarse de la carencia o déficits en la capacidad de discriminar los estados emocionales. La ausencia de referentes para los estados emocionales podría entenderse como consecuencia de la precariedad de experiencias importantes para ese aprendizaje (por ejemplo, ambientes familiares empobrecidos) y podrían dejar al sujeto con una experiencia somática indefinida frente a condiciones vitales emocionalmente activadoras. Pennebaker (1982) se refiere al mecanismo en cuestión del siguiente modo "los síntomas físicos están presentes sin la habilidad del paciente para evaluar que emociones -si es que alguna- pueden representar. Presumiblemente la persona no puede, es incapaz o no está dispuesta a atribuir los síntomas a la causa apropiada".

Finalmente, la adquisición predominante de repertorios conductuales característicos de los problemas somatoformes puede condicionar la trayectoria vital. Tal y como destacan Bass y Murphy (1995): "Las consecuencias del uso prolongado o recurrente del rol de enfermo como estrategia de afrontamiento es que fácilmente se afecta de modo adverso el estilo cognitivo, la autoimagen, la conducta interpersonal y otras áreas del desarrollo de la personalidad. La asociación de los trastornos somatoformes con los trastornos de la personalidad por evitación, pasivo-dependiente y otros atestigua el nexo con trayectorias desadaptativas en el desarrollo". 


\section{REFERENCIAS BIBLIOGRÁFICAS}

Allen, L., Escobar, J., Lehrer, P., Gara, M. y Woolfolk, R. L. (2002). Psychosocial treatments for multiple unexplained physical symptoms: a review of the literature. Psychosomatic Medicine, 64, 939-950.

Barsky, A. J. y Borus, J. F. (1995). Somatization and medicalization in the era of managed care. JAMA, 274, 24, 1931-1934.

Barsky, A. J. y Borus J. F. (1999). Functional somatic syndromes. Annals of Intemal Medicine, 130, 910-921.

Barsky, A. J., Coyetaux, R. R., Sarnie, M. K. y Cleary, P. D. (1993). Hypochondriacal patients" beliefs about good health. American Joumal of Psychiatry, 150, 7, 1085-1089.

Barsky, A. J., Ettner, S. L., Horsky, J. y Bates, D. W. (2001). Resource utilization of patients with hypochondriacal health anxiety and somatization. Medlcal Care, 39, 7, 705-715.

Bass, C. y Murphy, M. (1995). Somatoform and personality disorders: syndromal comorbility and overlapping developmental pathways. Joumal of Psychosomatic Research, 39, 4, 403-427.

Benjamin, S. y Eminson, D. M. (1992). Abnormal illness behaviour: Childhood experiences and long-term consequences. Intemational Joumal of Psychiatry, 4, 55-70.

Berrios, G E. (2001). Hypochondriasis: History of the concept. En Starcevic, V. y Lipsitt, D. R. (Ed). Hypochondriasis: Modern perspectives on an ancient malady. Oxford University Press.

Bhugra, D. y Mastogianni, A. (2004). Globalization and mental disorders. Overview with relation to depresión. British Joumal of Psychiatry, $184,1,10-20$.

Browlec, S., Leventhal, H. y Balaban, M. (1992). Autonomic correlates of illness imagery. Psychophysiology, 29, 2, 142-153.

Caballero, L. y Caballero, F. (2004). El paciente con somatizaciones crónicas. Psiquiatría y Atención Primaria, 5, 3, 4-11.

Ciechanowski, P. S., Walker, E. A., Katon, W. y Russo, J. E. (2002). Attachment theory: a model for health care utilization and somatization. Psychosomatic Medicine, 64, 660-667

Cloninger, R., Sigvardsson, S., Von Knorring, A. y Bohman, M. (1984). An adoptation study of somatoform disorders. I. Identification of two 
discrete somatoform disorders. Archives of General Psychiatry, 41, 863-871.

Costa, P. T. y McCrae, R. R. (1985). Hypochondriasis, neuroticism and aging. When are somatic complaints unfounded?. American Psychologist, 40, 1, 19-28.

Dantzer, R. (2005). Somatization: a psychoneuroinmune perspective. Psychoneuroendocrinology, 20, 1-6.

Epstein, R. M., Quill, T. E. y McWhinney, I. R. (1999). In Reply: more thoughts on somatization. Archives of Intemal Medicine, 159, 1814. Fink, P. (1996). Somatization: Beyond symptom count. Joumal of Psychosomatic Research, 40, 1, 7-10.

Friedberg, F. y Jason, L. A. (2001). Chronic fatigue syndrome and fibromyalgia: clinical assessment and treatment. Joumal of Clinical Psychology, 57, 4, 433-455.

Griffith, J. M., Griffith, M. E. y Slovik, L. S. (1989). Mind-body patterns of symptom generation. Family Practice, 28, 137-152.

Gureje, O. (2004). Why can we leam from a cross national study of somatic distress?. Joumal of Psychosomatic Research, 56, 409-412. Hayes, S., Wilson, K. y Strosahl, K. (1996). Experiential avoidance and behavioural disorders: a functional dimensional approach to diagnosis and treatment. Joumal of Consulting and Clinical 'Psychology, 64, 6, 1152-1168.

Horowitz, M. (1988). Introduction to psychodinamics. A new synthesis. Routledge.

Hotopf, M., Mayou, R., Wadsworth, M. y Wessely, S. (1999).

Psychosocial and developmental antecedents of chest pain in young adults. Psychosomatic Medicine, 61, 861-867.

Katon, W. J. y Walker, E. A. (1998). Medically unexplained symptoms in primary care. Joumal of Clinical Psychiatry, 59 (suppl. 20), 15-21. Katon, W., Lin, E., Von Korff, M., Russo, J., Lipscomb, P. y Buss, T. (1991). Somatization: a spectrum of severity. American Journal of Psychiatry, 148, 1, 34-40.

Katon, W., Ries, R. K. y Kleinman, A. (1984). the prevalence of somatization in primary care. Comprehensive Psychlatry, 25, 2, 208-215.

Katon, W., Sullivan, M. y Walker, E. (2001). Medical symptoms without identified pathology: relationship to psychiatric disorders, childhood 
and adult trauma and personality traits. Annals of Internal Medicine, 134, 917-925.

Katon, W., Kleinman, A. y Rosen, G. (1982). Depression and somatization: A review. The American Journal of Medicine, 72, 127-135.

Kellner, R. (1990). Somatization. Theories and research. The Joumal of Nervous and Mental Disease, 178, 3, 150-160.

Kirmayer, L., Groleau, D., Looper, K. y Dao, M. (2004). Explaining medically unexplained symptoms. Canadian Joumal of Psychiatry, 49, 10, 663-672.

Kirmayer, L. J. y Young, A. (1998). Culture and somatization: Clinical, epidemiological and ethnographic perspectives. Psychosomatic Medicine, 60, 420-430.

Kirmayer, L. J., Robbins, J. M: y Paris, J. (1994). Somatoform disorders: Personality and the social matrix of somatic distress. Journal of Abnormal Psychology, 103, 1, 125-136.

Kisely, S., Goldberg, D. y Simon, G. (1997). A comparison between somatic symptoms with and without clear organic cause: Results of an international study. Psychological Medicine, 27, 1011-1019. Kooiman, CG, Bolk JH, Rooijmans HG, Trijsburg RW. (2004). Alexithymia does not predict the persistence of medically unexplained physical symptoms. Psychosomatic Medicine, 66, 2, 224-32

Kowalski, R. M. (2002). Whining, griping and complaining: positivity and negativity. Joumal of Clinical Psychology, 58, 9, 1023-1035. Lesserman, J., Drossman, D. A., Zhiming, Tooley, T. C., Nachman, G. y Glogau, L. (1996). Sexual and physical abuse history in gastroenterology practice: how types of abuse impact health status. Psychosomatic Medicine, 58, 4-15.

Lieb, R., Zimmermann, P., Friis, R. H., Holler, M., Tholen, S. y Wittchen, H. U. (2002). The natural course of DSM-IV somatoform disorders and syndromes among adolescents and young adults: a prospectivelongitudinal community study. European Psychiatry, 17, 6, 321-31. Lim, S. y Kim, J. (2005). Cognitive processing of emotional information in depression, panic and somatoform disorder. Joumal of Abnormal Psychology, 114, 1, 50-61.

Lipowski, Z. J. (1988). Somatization: the concept and its clinical application. American Journal od Psychiatry, 145, 11, 1358-1367. 
Lobo, A., García Campayo, J., Campos, R., Marcos, G. y Echeverría, M. J. (1996). Somatization in Primary Care in Spain. I. Estimates of prevalence and clinical characteristics. British Journal of Psychiatry, 168, 344-353.

Looper, K. J. y Kirmayer, L. J. (2004). Perceived stigma in functional somatic symdromes and comparable medical conditions. Joumal of Psychosomatic Research, 57, 373-378.

Mayou, R. y Farmer, A. (2002). Functional somatic symptoms and syndromes. British Medical Joumal, 325, 265-268.

Murphy, M. (1989). Somatization: embodying the problem. The commonest way for psychiatric disorder to present. British Medical Joumal, 298, 1331-1332.

Overmier, J. (2002). Sensitization, conditioning and learning: can they help us understand somatization and disability. Scandinavian Joumal of Psychology, 43, 2, 105-112.

Papageorgiou, C. y Wells, A. (1998). Effects of attention training on hypochondriasis: a brief case series. Psychological Medicine, 28, 193-200.

Pennebaker, J. W. (1982). The Psychology of physical symptoms. New York, Springer

Pérez, M. (2004). Contingencia y Drama. Minerva Ediciones.

Raphael, K. G., Widom, C. y Lange, G. (2001). Childhood victimization and pain in adulthood: a prospective investigation. Pain, 92, 283-293. Reuber, M., Mitchell, A. J., Howlett, S. J., Crimlisl, H. L. y Grũnewald, R. A. (2005). Functional symptoms in Neurology: questions and answers. Joumal of Neurology, Neurosurgery and Psychiatry, 76, 307-314.

Rief, W. y Barsky, A. J. (2005). Psychobiological perspectives on somatoform disorders. Psychoneuroendocrinology, 20, 1-7. Salkovskis, P. M. (1988). Somatic Problems. En Hawton, K., Salkovskis, P. M., Kirk, J. y Clark, D. M., Cognitive-Behaviour therapy for psychiatric problems. Oxford University Press.

Salkovskis, P. M. y Warwick, H. (2001). Meaning, misinterpretations and medicine: A cognitive-behavioral approach to understand health anxiety and hypochondriasis. En En Starcevic, V. y Lipsitt, D. R. (Ed). Hypochondriasis: Modem perspectives on an ancient malady. Oxford University Press. 
Schmidt, S., Strauss, B. y Braehler, E. (2002). Subjetive physical complaints and hypochondriacal features from an attachment theoretical perspective. Psychology and Psychotherapy: Theory, Research and Practice, 75, 313-332.

Serlie, A. W., Duivenvoorden, H. J., Passchier, J., Ten Cate, F. J., Deckers, J. W. y Erdman, A. M. (1996). Empirical psychological modeling of chest pain: A comparative study. Journal of Psychosomatic Research, 40, 6, 625-635.

Speckens, A. E. M., Van Hemert, A. M., Bolk, J. H., Rooijmans, H. G.

M. y Hengeveld. (1996). Unexplained physical symptoms: outcome, utilization of medical care and associated factors. Psychological Medicine, 26, 745-752.

Staats, A. W. (1996/1997). Conducta y Personalidad. Conductismo Psicológico. Editorial Desclee de Brouwer.

Stone, J., Sharpe, M., Tothwell, P. M. y Warlow, C. P. (2003). The

12 year prognosis of unilateral functional weakness and sensory disturbance. Journal of Neurology, Neurosurgery and Psychiatry, 74, 591-596.

Stuart, S. y Noyes, R. (1999). Attachment and interpersonal comunication in somatization. Psychosomatics, 40, 1, 34-43

Torgersen, S. (1986). Genetics of somatoform disorders. Archives of General Psychiatry, 43, 502-505.

Van Houdenhove, B. y Egle, U. T. (2002). Letters to Editor: comment on Raphael, K. G., Widom, C. y Lange, G. (2001). Childhood victimization and pain in adulthood: a prospective investigation. Pain, 92, 283-293. Pain, 215-216.

Waitzkin, H. y Magaña, H. (1997). The black box of somatization: unexplained physical symptoms, culture and narratives of trauma. Social Science and Medicine, 45, 6, 811-825.

Watson, D. (1999). Dimensions underlying the anxiety disorders. Current Opinion in Psychiatry, 12, 181-186.

Williams, J. M. G., Stiles, W. B. y Shapiro, D. A. (1999). Cognitive mechanisms in the avoidance of painful and dangerous thoughts: elaborating the assimilation model. Cognitive Therapy and Research, 23, 3, 285-306.

Wilson, K. G y Luciano, M. C. (2002). Terapia de aceptación y compromiso. Un tratamiento conductual orientado a los valores. Ed. Pirámide. 